\title{
Pooled influenza vaccine effectiveness estimates for Australia, 2012-2014
}

\author{
S. G. SULLIVAN ${ }^{1,2,3,4 *}$, K. S. CARVILLE ${ }^{5}$, M. CHILVER ${ }^{3}$, J. E. FIELDING ${ }^{4,5,6}$,

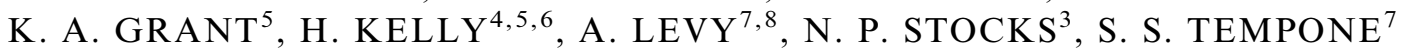 \\ AND A. K. REGAN ${ }^{8,9}$ \\ ${ }^{1}$ WHO Collaborating Centre for Reference and Research on Influenza at the Peter Doherty Institute for Infection and \\ Immunity, Melbourne, Victoria, Australia; ${ }^{2}$ Department of Epidemiology, University of California, Los Angeles, \\ California, USA; ${ }^{3}$ Discipline of General Practice, University of Adelaide, Adelaide, South Australia, Australia; ${ }^{4}$ School \\ of Population and Global Health, University of Melbourne, Victoria, Australia; ${ }^{5}$ Victorian Infectious Diseases Reference \\ Laboratory at the Peter Doherty Institute for Infection and Immunity, Melbourne, Victoria, Australia; \\ ${ }^{6}$ National Centre for Epidemiology and Public Health, Australian National University, Canberra, Australia; \\ ${ }^{7}$ PathWest Laboratory Medicine, Perth, Western Australia, Australia; ${ }^{8}$ School of Pathology and Laboratory \\ Medicine, University of Western Australia, Perth, Western Australia, Australia; ${ }^{9}$ Communicable Diseases \\ Control Directorate, Western Australian Department of Health, Perth, Western Australia, Australia
}

Received 30 August 2015; Final revision 2 March 2016; Accepted 6 April 2016;

first published online 29 April 2016

\section{SUMMARY}

Data were pooled from three Australian sentinel general practice influenza surveillance networks to estimate Australia-wide influenza vaccine coverage and effectiveness against community presentations for laboratory-confirmed influenza for the 2012, 2013 and 2014 seasons. Patients presenting with influenza-like illness at participating GP practices were swabbed and tested for influenza. The vaccination odds of patients testing positive were compared with patients testing negative to estimate influenza vaccine effectiveness (VE) by logistic regression, adjusting for age group, week of presentation and network. Pooling of data across Australia increased the sample size for estimation from a minimum of 684 to 3,683 in 2012, from 314 to 2,042 in 2013 and from 497 to 3,074 in 2014. Overall VE was 38\% [95\% confidence interval (CI) 24-49] in 2012, $60 \%(95 \%$ CI $45-70)$ in 2013 and $44 \%(95 \%$ CI 31-55) in 2014. For A(H1N1)pdm09 VE was $54 \%(95 \%$ CI-28 to 83$)$ in 2012, 59\% (95\% CI 33-74) in 2013 and 55\% (95\% CI 39-67) in 2014. For A(H3N2), VE was 30\% (95\% CI 14-44) in 2012, 67\% (95\% CI 39-82) in 2013 and $26 \%$ $(95 \%$ CI 1-45) in 2014. For influenza B, VE was stable across years at 56\% (95\% CI $37-70)$ in 2012, 57\% (95\% CI 30-73) in 2013 and 54\% (95\% CI 21-73) in 2014. Overall VE against influenza was low in 2012 and 2014 when A(H3N2) was the dominant strain and the vaccine was poorly matched. In contrast, overall VE was higher in 2013 when $\mathrm{A}(\mathrm{H} 1 \mathrm{~N} 1)$ pdm09 dominated and the vaccine was a better match. Pooling data can increase the sample available and enable more precise subtype- and age group-specific estimates, but limitations remain.

Key words: Influenza, influenza vaccine, influenza season, influenza-like illness, vaccine effectiveness.

\footnotetext{
* Author for correspondence: Dr S. G. Sullivan, WHO Collaborating Centre for Reference and Research on Influenza at the Peter Doherty Institute for Infection and Immunity, 792 Elizabeth Street, Melbourne, VIC 3000, Australia. (Email: sgsullivan@ucla.edu)
}

\section{INTRODUCTION}

Increasingly, it is recognized that annual estimates of influenza vaccine effectiveness (VE) are necessary given frequent changes to the vaccine composition as well as the circulating strains. In Australia, annual 
estimates for influenza VE using a general practitioner (GP) sentinel surveillance network have been published from Victoria, Australia, since 2009 [1]. However, until recently [2-5] these have been the only VE estimates regularly reported from the Southern Hemisphere. The generalizability of estimates from Victoria applied to the rest of Australia is unclear. A further problem with estimating VE from a single surveillance network is that there are often insufficient data generated to provide estimates by influenza type/subtype or age group. There may also be too few data for interim estimates. Thus, there is a need to increase the information available for calculating VE estimates for Australia.

Australia has three sentinel networks for influenzalike illness (ILI) surveillance in general practice. These are the Victorian Sentinel Practice Influenza Network (VicSPIN) [1], the Australian Sentinel Practices Research Network (ASPREN) [6, 7] and the Sentinel Practitioners Network of Western Australia (SPNWA) [3]. While VicSPIN and SPNWA perform surveillance for their respective states, ASPREN manages surveillance in the remaining six states and territories and also has several GPs in Victoria. VE estimates have previously been published from these networks for 2012 [2, 3, 8-10] and 2013 [11], but not 2014. In those analyses, samples were too small to enable estimates for specific age groups, such as children aged $<5$ years or the elderly. VE is expected to vary within age groups, given the varying level of exposure across the lifetime, the relative immaturity of the very young immune system [12] and immunosenescence in the elderly [13]. Thus there is a compelling need to estimate VE within age groups. Similarly, VE is expected to vary by influenza type and subtype [14]. Prior to 2015, influenza vaccines in Australia were all trivalent vaccines, containing an $\mathrm{A}(\mathrm{H} 3 \mathrm{~N} 2), \mathrm{A}(\mathrm{H} 1 \mathrm{~N} 1) \mathrm{pdm} 09$ and $\mathrm{B}$ component. So, while it is of public health interest to understand the overall performance of the vaccine, it is also helpful to estimate the effectiveness of each component.

The three Australian influenza surveillance networks use similar methods to collect information on patients presenting with ILI to sentinel GPs and can use the test-negative design to estimate VE [1-3]. These similarities mean the data can be easily pooled for estimation of nationwide VE. Pooling individual data across similarly designed studies has several advantages over individual reports or conventional meta-analyses of published estimates. First, the definitions and categorization of exposure, outcome and important confounders can be standardized [15]. Moreover, a uniform statistical model can be used, further eliminating analytical inconsistencies [16]. Variations in these parameters may be important sources of heterogeneity in studies of influenza VE [14]. Second, pooling data across studies increases the available sample size, which may permit subgroup analyses of the association of interest with greater statistical power than is possible in a single study [15]. In the case of influenza, pooling may permit estimation for groups which are typically under-represented in individual studies, such as young children and individuals with medical conditions, and importantly can enable evaluation of VE by subtype.

The purpose of the present study was to use data from three surveillance networks to calculate influenza VE estimates for all of Australia, as is regularly done in Europe [17], Canada [18] and the United States [19].

\section{METHODS}

\section{Study design}

Data for 2012, 2013 and 2014 from three influenza surveillance networks were used. Together, these networks included 256 GPs (ASPREN, 100; SPNWA, 64, VicSPIN, 92) in 2012, 262 GPs (ASPREN, 97; SPNWA, 71, VicSPIN, 94) in 2013 and 354 GPs (ASPREN, 177; SPNWA, 82, VicSPIN, 95) in 2014. Surveillance is year-round in ASPREN and SPNWA, but only from May to October (inclusive) in Victoria. Patients presenting with ILI (fever, cough, fatigue) were asked for nasal and throat swabs at the participating GPs' discretion. GPs collected demographic data (age, sex) and vaccination status. Since 2012, VicSPIN and SPNWA GPs additionally collected information about any comorbidities that could increase the risk of severe influenza, while ASPREN began collecting this information in 2014. Moreover, since 2012, VicSPIN collected patients' influenza status for the previous year, while ASPREN began collecting these data in 2014 and SPNWA does not collect this information.

Vaccination status was obtained via patient's medical record or patient's self-report. During 20122014 vaccines were produced by six manufacturers [20], but manufacturers' data were not collected in this study so all were assumed to have equal effectiveness. Vaccine components for each year are summarized in Table 1. Patients with an unknown vaccination status were excluded from the study. We 
Table 1. Patients' characteristics by vaccination status, 2012-2014

\begin{tabular}{|c|c|c|c|c|c|c|c|c|c|}
\hline & \multicolumn{3}{|l|}{2012} & \multicolumn{3}{|l|}{2013} & \multicolumn{3}{|l|}{2014} \\
\hline & \multicolumn{2}{|c|}{ Vaccination status } & \multirow[b]{2}{*}{$P$} & \multicolumn{2}{|c|}{ Vaccination status } & \multirow[b]{2}{*}{$P$} & \multicolumn{2}{|c|}{ Vaccination status } & \multirow[b]{2}{*}{$P$} \\
\hline & Unvaccinated & Vaccinated & & Unvaccinated & Vaccinated & & Unvaccinated & Vaccinated & \\
\hline Total & $2853(77)$ & $830(23)$ & & $1440(71)$ & $602(29)$ & & $2268(74)$ & $806(26)$ & \\
\hline \multicolumn{10}{|l|}{ Network } \\
\hline ASPREN & $1166(76)$ & $373(24)$ & & $763(70)$ & $321(30)$ & & $1159(72)$ & $450(28)$ & \\
\hline VicSPIN & $518(76)$ & $166(24)$ & & $228(73)$ & $86(27)$ & & $352(71)$ & $145(29)$ & \\
\hline SPNWA & $1169(80)$ & $291(20)$ & $0 \cdot 009$ & $449(70)$ & $195(30)$ & $0 \cdot 6$ & 757 (78) & $211(22)$ & $<0.01$ \\
\hline \multicolumn{10}{|l|}{ Gender } \\
\hline Female & $1388(74)$ & $486(26)$ & & $748(67)$ & $368(33)$ & & $1166(72)$ & $463(28)$ & \\
\hline Male & $1445(81)$ & $341(19)$ & $<0 \cdot 01$ & $688(75)$ & $232(25)$ & $<0.01$ & $1098(76)$ & $343(24)$ & $0 \cdot 004$ \\
\hline \multicolumn{10}{|l|}{ Age group, years } \\
\hline$<5$ & $366(95)$ & $21(5)$ & & $138(97)$ & $5(3)$ & & $249(95)$ & $13(5)$ & \\
\hline $5-19$ & $622(93)$ & $44(7)$ & & $269(91)$ & $27(9)$ & & $411(90)$ & $48(10)$ & \\
\hline $18-44$ & $1219(84)$ & $226(16)$ & & $653(77)$ & $195(23)$ & & $1012(80)$ & $248(20)$ & \\
\hline $45-64$ & $550(66)$ & $284(34)$ & & $332(61)$ & $214(39)$ & & $498(64)$ & $286(36)$ & \\
\hline$\geqslant 65$ & $96(27)$ & $255(73)$ & $<0.01$ & $48(23)$ & $161(77)$ & $<0 \cdot 01$ & $98(32)$ & $211(68)$ & $<0.01$ \\
\hline \multicolumn{10}{|l|}{ Influenza } \\
\hline Negative & $1645(74)$ & $576(26)$ & & $1068(67)$ & $533(33)$ & & $1561(72)$ & $622(28)$ & \\
\hline Positive & $1208(83)$ & $254(17)$ & $<0 \cdot 01$ & $372(84)$ & $69(16)$ & $<0.01$ & 707 (79) & $184(21)$ & $<0 \cdot 01$ \\
\hline \multicolumn{10}{|l|}{ Type/subtype } \\
\hline Negative & $1645(74)$ & $576(26)$ & & $1068(67)$ & $533(33)$ & & $1561(72)$ & $622(28)$ & \\
\hline $\mathrm{A}(\mathrm{H} 1 \mathrm{~N} 1) \mathrm{pdm} 09$ & $32(86)$ & $5(14)$ & & $135(84)$ & $25(16)$ & & $346(84)$ & $68(16)$ & \\
\hline $\mathrm{A}(\mathrm{H} 3 \mathrm{~N} 2)$ & $807(80)$ & $206(20)$ & & $83(84)$ & $16(16)$ & & $256(73)$ & $93(27)$ & \\
\hline B & $363(89)$ & $43(11)$ & & $148(85)$ & $26(15)$ & & $92(83)$ & $19(17)$ & \\
\hline A(NS) & $1(100)$ & $0(0)$ & & $5(71)$ & $2(29)$ & & $9(69)$ & $4(31)$ & \\
\hline Mixed & $5(100)$ & $0(0)$ & $<0 \cdot 01$ & $1(100)$ & $0(0)$ & $<0 \cdot 01$ & $4(100)$ & $0(0)$ & $<0 \cdot 01$ \\
\hline \multicolumn{10}{|l|}{ Haemagglutination inhibition result } \\
\hline A/California/7/2009-like*†: & $11(73)$ & $4(27)$ & & & & & & & \\
\hline A/California/7/2009-LR & $1(100)$ & $0(0)$ & & & & & & & \\
\hline A/Perth/16/2009-like* & $168(85)$ & $30(15)$ & & & & & & & \\
\hline A/Perth/16/2009-LR & $20(77)$ & $6(23)$ & & & & & & & \\
\hline A/Victoria/361/2011-like $\dagger$ & & & & $19(79)$ & $5(21)$ & & & & \\
\hline A/Switzerland/9 715 293/2013-liket & & & & & & & $55(75)$ & $18(25)$ & \\
\hline A/Switzerland/9 715 293/2013-LR & & & & & & & $5(83)$ & $1(17)$ & \\
\hline B/Brisbane/60/2008-like* & $68(94)$ & $4(6)$ & & & & & $1(100)$ & $0(0)$ & \\
\hline B/Brisbane/60/2008-LR & $13(93)$ & $1(7)$ & & & & & & & \\
\hline B/Wisconsin/1/2010-like $\dagger$ & $10(83)$ & $2(17)$ & & $4(80)$ & $1(20)$ & & & & \\
\hline
\end{tabular}




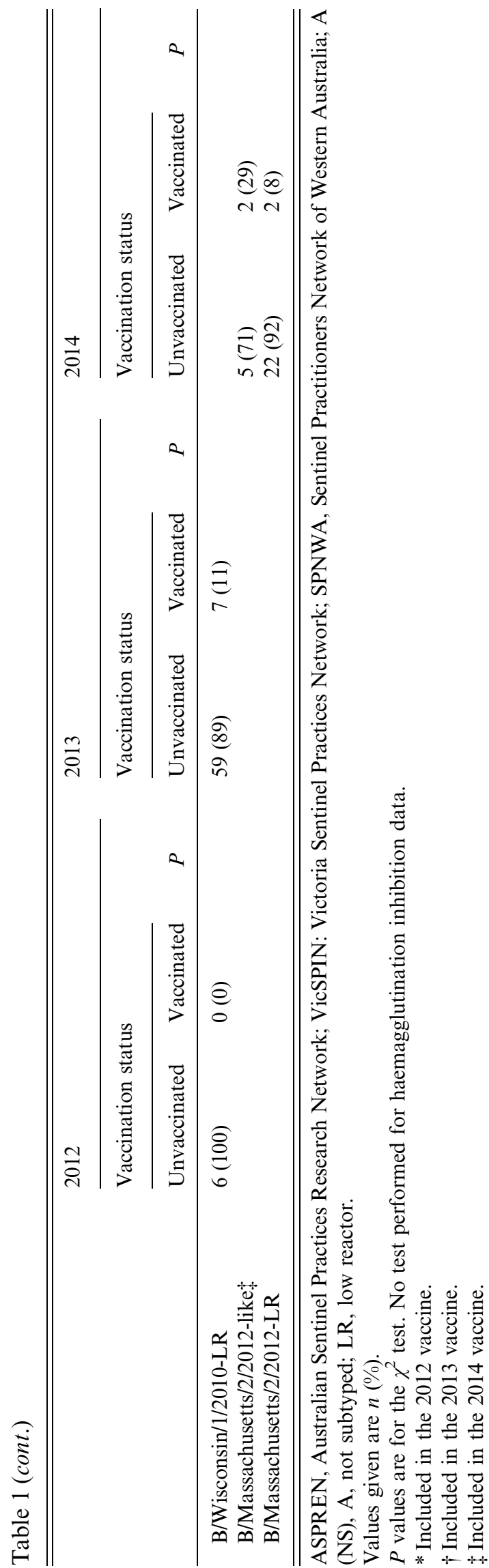

could not exclude patients on the basis of presenting too soon after vaccination, as the date of vaccination and date of onset were not collected by SPNWA or ASPREN. However, VicSPIN data suggested these were few in number.

\section{Laboratory methods}

Respiratory specimens were collected by GPs using preprepared kits. ASPREN doctors collected samples using Copan flocked swabs (Copan Diagnostics, USA) in $3 \mathrm{ml}$ universal transport medium (UTM), SPNWA doctors collected two nasal and one throat swab using Copan Mini Tip flocked swabs in virus transport medium and VicSPIN doctors used Copan flocked swabs in $3 \mathrm{ml}$ UTM. Samples were sent to SA Pathology (for ASPREN), PathWest Laboratory Medicine (for SPNWA) or the Victorian Infectious Diseases Reference Laboratory (VIDRL) (for VicSPIN) for testing. PathWest and VIDRL are national influenza centres, while SA Pathology is the public reference laboratory for the state of South Australia. Influenza was detected by real-time RT-PCR using in-house primers. Both the type and subtype were identified; however, SA Pathology only tested for A(H1N1) pdm09 and not $A(H 3 N 2)$ in 2012. Samples that were 'type A, not $\mathrm{A}(\mathrm{H} 1 \mathrm{~N} 1)$ pdm09' were treated as $\mathrm{A}$ (H3N2), based on virus characterization data available on a subset of these samples.

All influenza-positive specimens from VicSPIN, those with cycle threshold $\leqslant 30$ from ASPREN and those able to be isolated in culture at SPNWA were forwarded to the WHO Collaborating Centre for Reference and Research on Influenza in Melbourne where they were further characterized to identify the virus strain using the haemagglutination inhibition (HI) assay, as previously described [10, 21]. Isolates were identified as antigenically similar to the cell- or egg-propagated vaccine strain if the test samples had a titre that was $\leqslant$ fourfold different compared to the homologous vaccine reference strain. Results were reported against reference antisera raised against the vaccine strains.

\section{Statistical analysis}

All analyses were conducted in Stata version 12 (StataCorp., USA). Patients' characteristics by influenza status and vaccination status were compared by odds ratio (OR) and $\chi^{2}$ test for categorical variables. All $P$ values were two-sided. 

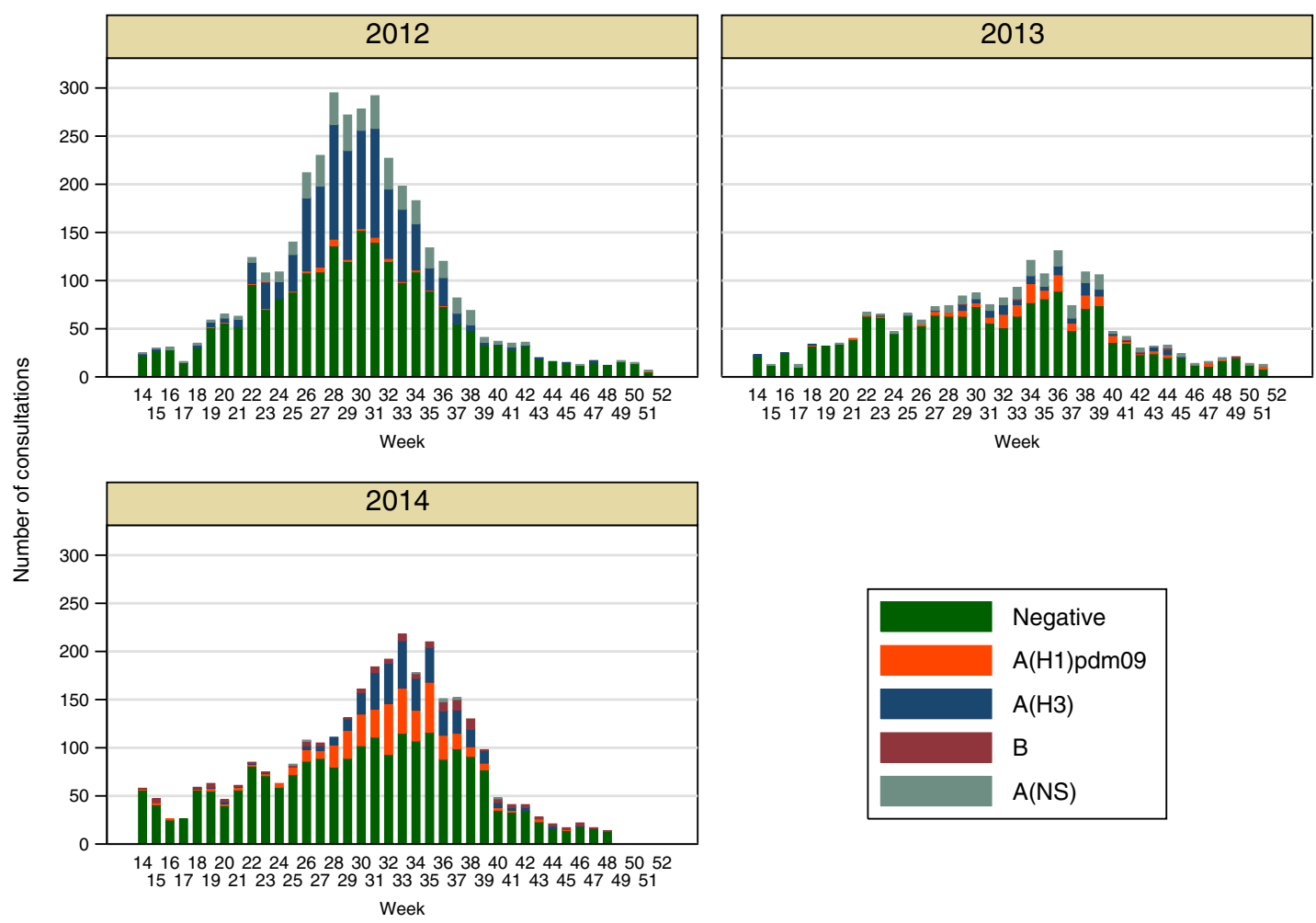

$141618202224262830323436 \quad 384042 \quad 444648 \quad 5052$
$15171921232527293133 \quad 353739414345474951$ Week

Fig. 1. Presentation of cases by influenza status.

To estimate VE, data were analysed using a testnegative design [22-24] where the exposure (vaccination) odds among those testing positive for influenza by RTPCR were compared to those testing negative; i.e. $\mathrm{VE}=$ $1-\mathrm{OR}_{\mathrm{adj}} \times 100 \%$. Estimates were adjusted for known confounders, selected a priori, including age group $(<5$, $5-17,18-64, \geqslant 65$ years), date of consultation modelled as a cubic spline with four knots, and network. Estimates were made for the periods of epidemic activity in each network. This period began when a positive case had been reported for two consecutive weeks at least 2 weeks after the annual vaccination campaign (mid-March) and ended after the peak when no case had been reported for at least 3 weeks. Prior to pooling, the data were meta-analysed, using the 'metan' command [25], to identify potential heterogeneity issues. The $I^{2}$ statistic was inspected and summary estimates were made using both fixed and random effects, where large discrepancies between the two were indicative of poor fit of the summary model $[26,27]$. The data were then pooled and modelled as described above, including a fixed effect for the network. Estimates were stratified by type/subtype as well as age group $(<18,18-64, \geqslant 65$ years). In a sensitivity analysis, data were multiply imputed using chained equations and VE estimated using the imputed data ( $n=20$ imputed datasets). Where there were fewer than four vaccinated cases (or unvaccinated cases) in an analysis or where the imputed model and the complete case model differed by $>10$ percentage points, the estimates were not reported because of potential sparse data bias [28].

\section{Ethical considerations}

ASPREN data were de-identified and obtained in accordance with National Health Security Act 2007. Therefore, Human Research Ethics Committee approval was not required. VicSPIN data were collected, used and reported under the legislative authorization of the Victorian Public Health and Wellbeing Act 2008 and Public Health and Wellbeing Regulations 2009 and thus did not require Human Research Ethics Committee approval. The SPNWA system is implemented by the Communicable Disease Control Directorate of the WA Department of Health as part of routine public health surveillance. Human Research Ethics Committee approval is not required.

\section{RESULTS}

Patients who consented to provide a swab sample for the surveillance networks in 2012, 2013 and 2014 


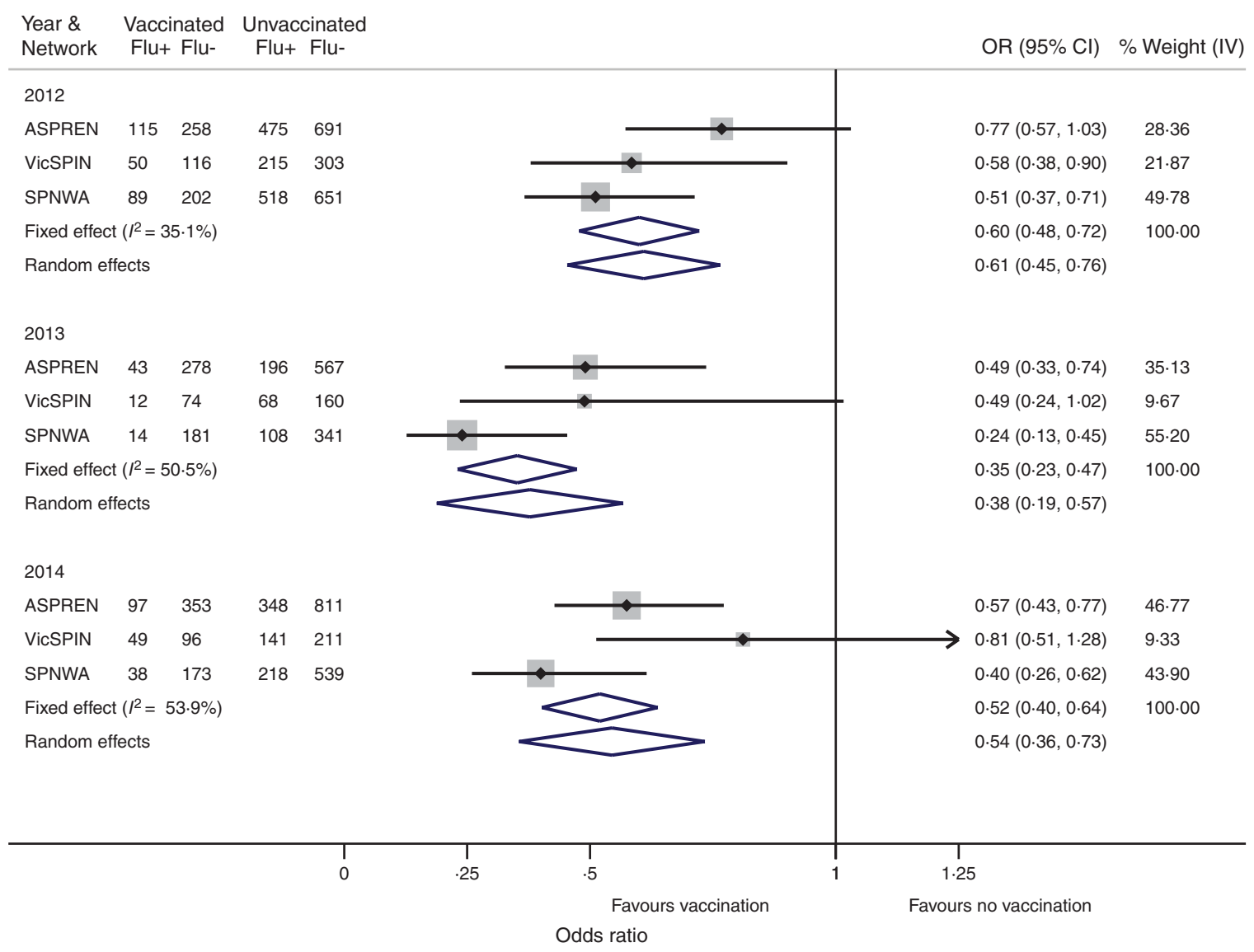

Fig. 2. Forest plots showing results of the initial exploration of heterogeneity by meta-analysis of unpooled data against all influenza types. Data for each year suggested moderate heterogeneity of around 35-54\% according to $I^{2}$. $I^{2}$ represents variation in vaccine effectiveness attributable to heterogeneity. This statistic measures overlap between the confidence intervals and point estimates among studies. For example, the heterogeneity in 2012 has resulted from failure of the confidence interval of ASPREN to overlap the point estimate of SPNWA (and vice versa). Random-effects model from the DerSimonian \& Laird method [25]. Fixed-effects estimate from the Mantel-Haenszel method. Cochran's $P$ value not shown as it is underpowered. ASPREN, Australian Sentinel Practices Research Network; VicSPIN, Victorian Sentinel Practice Influenza Network; SPNWA, Sentinel Practitioners Network of Western Australia.

numbered 4115, 2371 and 3570, respectively. Of these, 432, 329 and 496 were excluded (see Supplementary Table S1). In 2012, ILI presentations peaked in week 28 and continued until week 51 (Fig. 1). In contrast, presentations in 2013 peaked much later in week 36 and continued until the end of the year. In 2014, presentations peaked in week 33 and continued until week 48.

Patient characteristics are shown by vaccination status in Table 1 and by influenza status in Table 2. The 2012 season was characterized by a higher percentage of positive tests $(40 \%)$ than $2013(22 \%)$ or 2014 $(29 \%)$, and the dominant virus was $\mathrm{A}(\mathrm{H} 3 \mathrm{~N} 2)(69 \%$ of confirmed influenza cases) (Table 2). In 2013, A $(\mathrm{H} 3 \mathrm{~N} 2)$ viruses were least frequent $(22 \%)$ and the majority of viruses (39\%) were influenza B, followed closely by A(H1N1)pdm09 (36\%). In 2014, A(H1N1)pdm09 was dominant (46\%), followed by $\mathrm{A}(\mathrm{H} 3 \mathrm{~N} 2)(39 \%)$. HI assays suggested that most viruses were antigenically similar to their relevant influenza A vaccine strain (Table 1), but in 2013 and 2014 were poorly matched to the influenza B strain. In addition, in 2013 and 2014, A(H3N2) viruses showed good antigenic match to the cell-propagated strain (Table 1) but all were low reactors to the egg-propagated strain (data not shown).

The proportion of vaccinated patients ranged from $23 \%$ in 2012 to $29 \%$ in 2013 and was generally higher in women than men (Table 1). In addition, vaccine coverage increased with increasing age, being very low in young children ( $<5$ years) at $3-5 \%$ and relatively high in the elderly ( $>65$ years) at $68-77 \%$.

Preliminary analysis indicated moderate heterogeneity, with $I^{2}$ ranging from $35 \%$ in 2012 to $54 \%$ in 2014 (Fig. 2). The fixed- and random-effects estimates were 

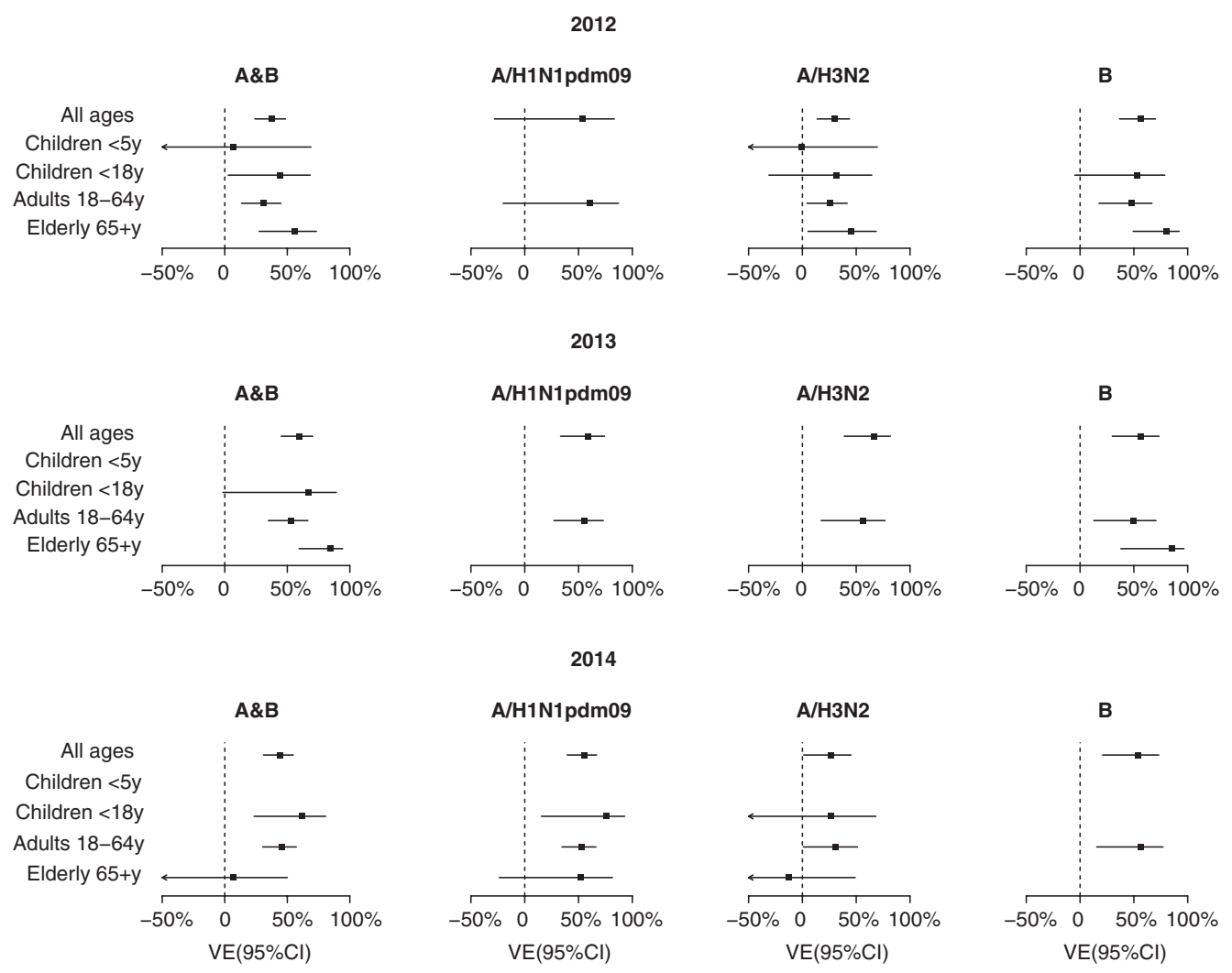

Fig. 3. Vaccine effectiveness and 95\% confidence interval [VE (95\% CI)] estimates by age group within types/subtypes.

consistent and did not highlight any major heterogeneity problems. Based on these two metrics, data were pooled for VE estimation. VE estimates for each age group with type/subtype are presented in Figure 3. Estimates and their sample sizes are shown in Supplementary Table S2. VE estimates obtained using the imputed data were generally within a percentage point of the complete-case analysis. Thus, they did not suggest any substantial bias in the complete-case analysis.

VE for any type of influenza for all patients was $38 \%$ [95\% confidence interval (CI) 24-49] in 2012, 60\% (95\% CI 45-70) in 2013 and 44\% (95\% CI 31-55) in 2014. For influenza A(H1N1)pdm09, VE estimates were similar across the seasons studied at 54\% (95\% CI -28 to 83 ) in $2012,59 \%$ (95\% CI 33-74) in 2013 and $55 \%$ (95\% CI 39-67) in 2014. There were insufficient data available to make estimates for this subtype for children or the elderly in 2012 or 2013.

For influenza $\mathrm{A}(\mathrm{H} 3 \mathrm{~N} 2), \mathrm{VE}$ for all age groups was low in 2012 at $30 \%(95 \%$ CI 14-44) and in 2014 at $26 \%$ (95\% CI 1-45), but higher in 2013 at $67 \%$ (95\% CI 39-82). Point estimates were lowest for the elderly in 2014 and highest for working-age adults in 2013. There were too few data to make a reliable estimate for the elderly or children in 2013, nor were there sufficient data to make estimates for children aged $<5$ years in 2014.

For influenza B, VE point estimates were similar in all years, despite the change in dominant lineage. Estimates for all ages were 56\% (95\% CI 37-70) in $2012,57 \%(95 \%$ CI 30-73) in 2013 and 54\% $(95 \%$ CI 21-73) in 2014. There were too few vaccinated cases to estimate VE for children aged $<5$ years in any year, or for children aged $<18$ years in 2013 and 2014 , and there were too few unvaccinated cases to estimate VE in the elderly in 2014.

\section{DISCUSSION}

In this study, data from three influenza surveillance schemes were pooled to increase the sample used and enable estimation of influenza VE within types/ subtypes and age groups. This permitted estimation of $\mathrm{VE}$ for $\mathrm{A}(\mathrm{H} 1 \mathrm{~N} 1) \mathrm{pdm} 09$ in 2012, which had not previously been possible using only the VicSPIN or ASPREN data $[2,10]$ and permitted type-/subtypespecific estimation within some age groups, which had not previously been reported for the years studied $[2,3,10,11]$. In 2012, the VE estimates reported by these three networks were $23 \%(95 \% \mathrm{CI}-4$ to 43$)$ 
for ASPREN, 45\% (95\% CI 8-66) for VicSPIN, and $49 \%$ (95\% CI 30-63) for SPNWA, while the summary estimate reported in this study was within this range at $38 \%$ (95\% CI $24-49)$. The pooled estimate was within the range of the previous estimate and had higher precision (see Supplementary Table S3). For other comparisons, only an $\mathrm{A}(\mathrm{H} 3 \mathrm{~N} 2)$ estimate was previously reported by all these networks in 2012. Again, the pooled estimate fell within the range of the published estimates. For other subtypes and for age groups, only SPNWA and ASPREN were able to report previously. Published estimates were similar to the pooled estimates for influenza B and for the elderly. For children aged $<18$ years and adults, the pooled estimate fell between the two published estimates. It should be noted that the statistical model used, the exclusion criteria applied and the statistical power in the published studies varied. Exploration of heterogeneity for the overall estimates revealed moderate heterogeneity. Heterogeneity was not explored within types/subtypes and may have been greater in these subgroups given the reduced sample size. However, this may not be reflected in the metrics used to detect heterogeneity, which would have reduced power in subgroup analysis.

Our VE estimates compared variably with those reported elsewhere. For example, our VE estimate for $\mathrm{A}(\mathrm{H} 1 \mathrm{~N} 1) \mathrm{pdm} 09$ was $54 \%(95 \% \mathrm{CI}-28$ to 83$)$ in 2012, which was lower than estimates reported from the United States (VE 65\%, 95\% CI 44-79) [19] and Canada (VE 80\%, 95\% CI 52-92) [18]. Our 2013 estimate for $\mathrm{A}(\mathrm{H} 1 \mathrm{~N} 1) \mathrm{pdm} 09$ was $59 \%$ (95\% CI 33-74) and not substantially different from New Zealand's (49\%, 95\% CI -90 to 86$)$ [4], Europe's (50\%, 95\% CI 28-66) [29] or Canada's (59\%, 95\% CI 16-80\%) [30]. The 2014 estimate of $55 \%$ (95\% CI 39-67) was close to New Zealand's (VE 59\%, 95\% CI 36-74) [31] and Greece's (VE 57\%, 95\% CI 23-76) [32]. For A(H3N2), our 2012 estimate was 30\% (95\% CI 14 44), and similar to the UK's estimate (VE 26\%, 95\% CI -4 to 48) [33] and Canada's (VE 41\%, 95\% CI 17-59). The 2013 estimate at $67 \%(95 \%$ CI 39-82) was similar to the estimate from New Zealand (VE $61 \%, 95 \%$ CI 32-77), but higher than several estimates from the Northern Hemisphere 2012/2013 season [29, 33, 34]. The $2014 \mathrm{~A}(\mathrm{H} 3 \mathrm{~N} 2)$ estimate was low (VE $26 \%, 95 \%$ CI 1-45), which was in line with other reports for that season $[31,35]$. In all years, most point estimates for influenza $\mathrm{B}$, including ours, were around $50-60 \%[4,19,29,31,33,36]$.

Despite pooling, there remained too few cases for some of the analyses attempted. For example, vaccination coverage was too low in young children aged $<5$ years to permit estimation for $\mathrm{A}(\mathrm{H} 1 \mathrm{~N} 1)$ pdm09 in 2012 or for any influenza type/subtype in 2013 or 2014. Vaccination coverage in young children in Australia is generally very low. In Western Australia, the government began subsidising influenza vaccination for children aged $<5$ years in 2008 , which substantially increased uptake; surveillance data indicated uptake around 50\% in 2008-2009 [37]. However, serious adverse reactions to the 2010 vaccine in some children led to a loss of consumer confidence [38], and in 2012 only $10 \cdot 1 \%$ of children were vaccinated, half of whom were only partially vaccinated [37]. At such low coverage, the power to see a modest effect (e.g. $50 \%$ ) is extremely limited and a sample consisting of at least 365 influenza-positive cases is required to gain statistical significance (assuming a case-control ratio of 3:1, at $\alpha=0 \cdot 05, \beta=0 \cdot 2$ ). Infections in children are proportionally more common than infections in adults and the health and economic costs of infection in this group are significant [39-43]. However, reliable information on the effectiveness of the inactivated vaccine in children is scant [44]. In this study, despite pooling data across networks estimation of type-/ subtype-specific VE in young children was not possible. Thus, there is a compelling need to scale up influenza surveillance activities in children and other groups with low vaccination uptake.

There were also insufficient cases of $\mathrm{A}(\mathrm{H} 1 \mathrm{~N} 1)$ pdm09 in the elderly to permit estimation. Low A (H1N1)pdm09 infection rates in the elderly have been reported since the pandemic in 2009 [45], a phenomenon attributed to prior infection with a similar influenza strain many years earlier [46, 47]. In contrast, $\mathrm{A}(\mathrm{H} 3 \mathrm{~N} 2)$ viruses tend to exhibit more rapid antigenic and genetic drift than $\mathrm{A}(\mathrm{H} 1 \mathrm{~N} 1)$ pdm09 viruses [48, 49], resulting in continued vulnerability throughout an individual's lifetime. Indeed, in this study the proportion of elderly patients with A (H3N2) in 2012 was similar to the overall proportion of patients testing positive for $\mathrm{A}(\mathrm{H} 3 \mathrm{~N} 2)$ and that for children, at around $30 \%$. For $\mathrm{A}(\mathrm{H} 3 \mathrm{~N} 2)$, VE was modest in the elderly in 2012 and was not estimated in 2013 due to the low number of vaccinated cases, again highlighting that pooling has not adequately overcome sample size limitations for subtype-/age group-specific estimates using current surveillance programmes in Australia.

Despite the change to the B lineage included in the vaccine between 2012 and 2013 (i.e. from Victoria to Yamagata), VE point estimates were the same. In 
contrast, VE against A(H3N2) was poor in 2012 when $\mathrm{A}(\mathrm{H} 3 \mathrm{~N} 2)$ dominated and most viruses were antigenically similar to the vaccine strain, A/Perth/16/2009. Estimates were paradoxically moderate in 2013 when all isolated $\mathrm{A}(\mathrm{H} 3 \mathrm{~N} 2)$ viruses were low reactors to the egg-propagated reference strain. Egg-acquired adaptations in the vaccine's $\mathrm{A}(\mathrm{H} 3 \mathrm{~N} 2)$ strain resulted in poor VE globally in 2012 [30], so VE was expected to be similarly low in Australia in 2013 and 2014. Genetic analysis was not routinely performed in these networks, so it was not possible to perform a thorough examination of clade variation that may have explained the relatively high VE for influenza $\mathrm{A}(\mathrm{H} 3 \mathrm{~N} 2)$ in 2013 compared to Northern Hemisphere estimates for the previous and following seasons. Limited genetic information from Victoria in 2012 [10] suggested about half of viruses fell into a genetic clade that differed from the vaccine clade, but no clustering by vaccination status was observed. Increasingly, evidence is surfacing that both antigenic and genetic matches between the vaccine and circulating strains correlates poorly with VE estimates and may vary with the virus type/subtype $[10,50,51]$. Great efforts are being made to explore alternative options for measuring antigenic match [52].

This study had several limitations. First, the decision to pool was made retrospectively, so the data collection instruments did not collect exactly the same information across networks. For example, not all networks collected the date of vaccination so we were unable to exclude patients who presented too soon after vaccination, and who may have been misclassified as vaccinated. Moreover, how vaccination status was ascertained was not recorded and in many cases may have been by self-report. This may be an underappreciated source of measurement error in studies of influenza VE [14]. Study coordinators are investigating the use of abstracting vaccination status and testing results from GP practice software for the purpose of measuring VE [53]. Second, the date of symptom onset was also not routinely collected, so we were unable to remove patients presenting too late after onset. GPs were instructed to only sample patients presenting within 4 days of illness onset, but study coordinators are aware that this stipulation is not strictly adhered to. Exclusion of patients who present too late can have quite an impact on VE point estimates [14]. In theory, non-differential misclassification of outcome status due to poor sensitivity should only minimally bias estimates [14]. This has been the case in VicSPIN, where applying a restriction reduced adjusted VE estimates by $7-15 \%$ in 2007 [54],
$5-35 \%$ in 2008 [54], $0-3 \%$ in 2010 [55], 2-14\% in 2011 [56] and 7\% in 2013 [11]. Third, we were unable to adjust for the presence of comorbidities which might increase a person's likelihood of vaccination and infection. Although this information was collected by all networks in 2014, collection was inconsistent and precluded its use. In any case, interim estimates from Canada in 2013 and 2014 suggested that the inclusion of a variable indicating the presence of comorbid conditions resulted in minimal changes to point estimates and confidence intervals [57, 58]. Moreover, many conditions included in this category affect susceptibility to a severe infection, but not infection itself and thus do not fulfil the conditions for confounding.

We did not combine estimates across the seasons studied. Figure 1 clearly shows that the severity of the season and predominant strains for each season differed substantially, so the outcomes of interest differed across seasons. Moreover, there were changes made to the vaccine between 2012 and 2014, so the exposure of interest also differed by season. Such differences are the justification for estimating VE each season, and this rationale should be equally applied to the pooling of VE estimates. While meta-analyses have reported summary estimates that combine data across seasons $[59,60]$, this may not be sensible practice. Similarly, although reported here, there may be limited value in reporting an overall VE estimate combining data for $\mathrm{A}(\mathrm{H} 1 \mathrm{~N} 1) \mathrm{pdm} 09, \mathrm{~A}(\mathrm{H} 3 \mathrm{~N} 2)$ and $\mathrm{B}$ viruses. There is substantial evidence that $\mathrm{VE}$ varies by type/subtype [14], and the estimation of an overall effect masks problems with the vaccine, particularly for the $A(H 3 N 2)$ viruses [30]. However, this is often the estimate of interest to public health practitioners and provides a single estimate comparable across seasons.

Analogously, it could be argued that combining data across geographically disparate areas could result in too much heterogeneity to enable reliable estimation of effect. This might be particularly true if there are latitudinal variations. Australia experiences a range of climates, from tropical to temperate to alpine. The epidemic period can vary between such climates [61-63], and in Northern Australia, there is often influenza activity around March, far earlier than the winter season. However, the population density in the far north of Australia is low. There were only six SPNWA and 11 ASPREN GPs obtaining samples in tropical regions. In addition, the main epidemic period in the Northern Territory tends to coincide with that of 
Table 2. Patients' characteristics by influenza status, 2012-2014

\begin{tabular}{|c|c|c|c|c|c|c|c|c|c|}
\hline & \multicolumn{3}{|l|}{2012} & \multicolumn{3}{|l|}{2013} & \multicolumn{3}{|l|}{2014} \\
\hline & \multicolumn{2}{|c|}{ Influenza status } & \multirow[b]{2}{*}{$P$} & \multicolumn{2}{|c|}{ Influenza status } & \multirow[b]{2}{*}{$P$} & \multicolumn{2}{|c|}{ Influenza status } & \multirow[b]{2}{*}{$P$} \\
\hline & Negative & Positive & & Negative & Positive & & Negative & Positive & \\
\hline Total & $2221(60)$ & $1462(40)$ & & $1601(78)$ & $441(22)$ & & $2183(71)$ & $891(29)$ & \\
\hline \multicolumn{10}{|l|}{ Network } \\
\hline ASPREN & $949(62)$ & $590(38)$ & & $845(78)$ & $239(22)$ & & $1164(72)$ & $445(28)$ & \\
\hline VicSPIN & $419(61)$ & $265(39)$ & & $234(75)$ & $80(25)$ & & 307 (62) & $190(38)$ & \\
\hline SPNWA & $853(58)$ & $607(42)$ & $0 \cdot 2$ & $522(81)$ & $122(19)$ & $0 \cdot 06$ & $712(74)$ & $256(26)$ & $<0.01$ \\
\hline \multicolumn{10}{|l|}{ Gender } \\
\hline Female & $1154(62)$ & $720(38)$ & & $888(80)$ & $228(20)$ & & $1172(72)$ & $457(28)$ & \\
\hline Male & $1056(59)$ & $730(41)$ & $0 \cdot 1$ & 709 (77) & $211(23)$ & $0 \cdot 2$ & $1008(70)$ & $433(30)$ & $0 \cdot 2$ \\
\hline \multicolumn{10}{|l|}{ Age group, years } \\
\hline$<5$ & $266(69)$ & $121(31)$ & & $124(87)$ & $19(13)$ & & $208(79)$ & $54(21)$ & \\
\hline $5-19$ & $277(42)$ & $389(58)$ & & $199(67)$ & $97(33)$ & & $288(63)$ & $171(37)$ & \\
\hline $18-44$ & $901(62)$ & $544(38)$ & & $654(77)$ & $194(23)$ & & $893(71)$ & 367 (29) & \\
\hline 45-64 & $553(66)$ & $281(34)$ & & $439(80)$ & $107(20)$ & & $554(71)$ & $230(29)$ & \\
\hline$\geqslant 65$ & $224(64)$ & $127(36)$ & $<0 \cdot 01$ & $185(89)$ & $24(11)$ & $<0.01$ & $240(78)$ & $69(22)$ & $<0 \cdot 01$ \\
\hline \multicolumn{10}{|l|}{ Vaccination status } \\
\hline Unvaccinated & $1645(58)$ & $1208(42)$ & & $1068(74)$ & $372(26)$ & & $1561(69)$ & $707(31)$ & \\
\hline Vaccinated & $576(69)$ & $254(31)$ & $<0 \cdot 01$ & $533(89)$ & $69(11)$ & $<0.01$ & $622(77)$ & $184(23)$ & $<0.01$ \\
\hline
\end{tabular}

ASPREN, Australian Sentinel Practices Research Network; VicSPIN, Victoria Sentinel Practices Network; SPNWA, Sentinel Practitioners Network of Western Australia; Values given are $n(\%)$.

southern, temperate regions of the country [64]. Finally, cases from March would be excluded in this analysis as they precede or coincide with the roll-out of the vaccine. If people infected in March were then more likely to get vaccinated, it could inflate VE estimates, because their natural immunity would protect them, not the vaccine. However, because their populations are small, it is unlikely these geographical variations will have influenced the results here.

In summary, pooling of Australia-wide data enabled estimation of subtype-specific VE estimates, although there continued to be insufficient sample for some age group-specific subtype estimates. Increased study power arising from pooling of data may eventually mean other VE strata can be considered, such as vaccine brand, which is not routinely collected by any of the three networks but may be possible to obtain through abstraction of data from GP practice software and the recent establishment of an adult vaccination register in Australia. Data pooling may also prove useful for improving the precision of interim VE estimates. Australia currently does not routinely publish interim VE estimates, but does contribute data to the vaccine strain selection meeting [52]. Harmonization of the data collected by the networks has already begun which will also enable better integration of the data, reduce residual heterogeneity, and permit adjustment for variables, such as comorbidity status.

\section{SUPPLEMENTARY MATERIAL}

For supplementary material accompanying this paper visit http://dx.doi.org/10.1017/S0950268816000819.

\section{ACKNOWLEDGEMENTS}

We gratefully acknowledge all practices and general practitioners who participated in sentinel surveillance. We thank the laboratory staff of the SA Pathology, PathWest, the Victorian Infectious Diseases Reference Laboratory, and the WHO Collaborating Centre for Reference and Research on Influenza. Thanks also to Shuo Feng for assistance with Figure 3.

The Australian Sentinel Practices Research Network and the Melbourne WHO Collaborating Centre for Reference and Research on Influenza are supported by the Australian Government Department of Health. The Sentinel Practitioners Network of Western Australia is funded by the Western Australian Department of Health. The Victorian Sentinel Practice Influenza Network is funded by the Victorian Government Department of Health and Human Services. 


\section{DECLARATION OF INTEREST}

None.

\section{REFERENCES}

1. Kelly $\mathbf{H}$, et al. Estimation of influenza vaccine effectiveness from routine surveillance data. PLoS ONE 2009; 4: e5079.

2. Sullivan SG, et al. Influenza vaccine effectiveness in Australia: results from the Australian Sentinel Practices Research Network. Medical Journal of Australia 2014; 201: 109-111.

3. Levy A, et al. Influenza vaccine effectiveness estimates for Western Australia during a period of vaccine and virus strain stability, 2010 to 2012. Vaccine 2014; 32: 6312-6318.

4. Turner N, et al. Effectiveness of seasonal trivalent inactivated influenza vaccine in preventing influenza hospitalisations and primary care visits in Auckland, New Zealand, in 2013. Eurosurveillance 2014; 19.

5. McAnerney JM, et al. Effectiveness and knowledge, attitudes and practises of seasonal influenza vaccine in primary health care settings in South Africa, 20102013. Influenza and Other Respiratory Viruses 2015; 9: $143-150$.

6. Anon. Surveillance systems reported in CDI, 2011. Communicable Diseases Intelligence Quarterly Report 2011; 35: 55-59.

7. Parrella A, et al. ASPREN surveillance system for influenza-like illness - a comparison with FluTracking and the National Notifiable Diseases Surveillance System. Australian Family Physician 2009; 38: 932-936.

8. Sullivan SG, Tay EL, Kelly H. Variable definitions of the influenza season and their impact on vaccine effectiveness estimates. Vaccine 2013; 31: 4280-4283.

9. Sullivan SG, Kelly H. Late season interim estimates of influenza vaccine effectiveness reliably predict end of season estimates in Victoria, Australia, 2007-2012. Eurosurveillance 2013; 18: pii $=20605$.

10. Sullivan SG, et al. Influenza vaccine effectiveness during the 2012 influenza season in Victoria, Australia: influences of waning immunity and vaccine match. Journal of Medical Virology 2014; 86: 1017-1025.

11. Carville KS, et al. Understanding influenza vaccine protection in the community: an assessment of the 2013 influenza season in Victoria, Australia. Vaccine 2015; 33: $341-345$.

12. Widdowson MA, Monto AS. Epidemiology of influenza. In: Wenster RG, et al., eds. Textbook of Influenza, 2nd edn. Chichester, West Sussex, UK: Wiley Blackwell, 2013, pp. 254-255.

13. Haq K, McElhaney JE. Immunosenescence: influenza vaccination and the elderly. Current Opinions in Immunology 2014; 29: 38-42.

14. Sullivan SG, Feng S, Cowling BJ. Potential of the testnegative design for measuring influenza vaccine effectiveness: a systematic review. Expert Review of Vaccines 2014; 13: 1571-1591.
15. Smith-Warner SA, et al. Methods for pooling results of epidemiologic studies: the Pooling Project of Prospective Studies of Diet and Cancer. American Journal of Epidemiology 2006; 163: 1053-1064.

16. Blettner M, Sauerbrei W. Influence of model-building strategies on the results of a case-control study. Statistics in Medicine 1993; 12: 1325-1338.

17. Kissling E, et al. 'I-MOVE' towards monitoring seasonal and pandemic influenza vaccine effectiveness: lessons learnt from a pilot multi-centric case-control study in Europe, 2008-9. Euroaurveillance 2009; 14.

18. Skowronski DM, et al. Influenza A/subtype and B/lineage effectiveness estimates for the 2011-2012 trivalent vaccine: cross-season and cross-lineage protection with unchanged vaccine. Journal of Infectious Diseases 2014; 210: 126-137.

19. Ohmit SE, et al. Influenza vaccine effectiveness in the 2011-2012 season: protection against each circulating virus and the effect of prior vaccination on estimates. Clinical Infectious Diseases 2014; 58: 319-327.

20. Australian Government Department of Health and Ageing. Part 4: Vaccine-preventable diseases. In: The Australian Immunisation Handbook, 10th edn. Canberra: National Health and Medical Research Council, 2015, pp. 251-256.

21. Hobson D, et al. The role of serum haemagglutination-inhibiting antibody in protection against challenge infection with influenza A2 and B viruses. Journal of Hygiene 1972; 70: 767-777.

22. Jackson ML, Nelson JC. The test-negative design for estimating influenza vaccine effectiveness. Vaccine 2013; 31: $2165-2168$.

23. Orenstein EW, et al. Methodologic issues regarding the use of three observational study designs to assess influenza vaccine effectiveness. International Journal of Epidemiology 2007; 36: 623-631.

24. Skowronski D, et al. Effectiveness of vaccine against medical consultation due to laboratory-confirmed influenza: results from a sentinel physician pilot project in British Columbia, 2004-2005. Canada Communicable Disease Report 2005; 31: 181-191.

25. Harris R, et al. metan: fixed- and random-effects meta-analysis. Stata Journal 2008; 8: 3-28.

26. Greenland $\mathbf{S}$. Invited commentary: a critical look at some popular meta-analytic methods. American Journal of Epidemiology 1994; 140: 290-296.

27. Friedenreich CM. Methods for pooled analyses of epidemiologic studies. Epidemiology 1993; 4: 295-302.

28. Greenland S. Bayesian perspectives for epidemiological research. II. Regression analysis. International Journal of Epidemiology 2007; 36: 195-202.

29. Kissling E, et al. Influenza vaccine effectiveness estimates in Europe in a season with three influenza type/ subtypes circulating: the I-MOVE multicentre casecontrol study, influenza season 2012/13. Eurosurveillance 2014; 19.

30. Skowronski DM, et al. Low 2012-13 influenza vaccine effectiveness associated with mutation in the eggadapted $\mathrm{H} 3 \mathrm{~N} 2$ vaccine strain not antigenic drift in circulating viruses. PLOS ONE 2014; 9: e92153. 
31. Pierse N, et al. Influenza vaccine effectiveness for hospital and community patients using control groups with and without non-influenza respiratory viruses detected, Auckland, New Zealand 2014. Vaccine 2016; 34: 503-509.

32. Lytras $\mathbf{T}$, et al. Influenza vaccine effectiveness against laboratory confirmed influenza in Greece during the 2013-2014 season: a test-negative study. Vaccine 2015; 33: $367-373$.

33. Andrews $\mathbf{N}$, et al. Effectiveness of trivalent seasonal influenza vaccine in preventing laboratory-confirmed influenza in primary care in the United Kingdom: 2012/ 13 end of season results. Eurosurveillance 2014; 19: 5-13.

34. Yang $\mathbf{P}$, et al. Influenza vaccine effectiveness against medically-attended influenza illness during the 20122013 season in Beijing, China. Vaccine 2014; 32: 5285-5289.

35. Jimenez-Jorge $\mathbf{S}$, et al. Interim influenza vaccine effectiveness: A good proxy for final estimates in Spain in the seasons 2010-2014. Vaccine 2015; 33: 3276-3280.

36. Castilla $\mathbf{J}$, et al. Decline in influenza vaccine effectiveness with time after vaccination, Navarre, Spain, season 2011/12. Eurosurveillance 2013; 18: pii $=20388$.

37. Blyth CC, et al. Effectiveness of trivalent flu vaccine in healthy young children. Pediatrics 2014; 133: e1218-1225.

38. Kelly HA, et al. Adverse events associated with 2010 CSL and other inactivated influenza vaccines. Medical Journal of Australia 2011; 195: 318-320.

39. Ampofo K, et al. Epidemiology, complications, and cost of hospitalization in children with laboratoryconfirmed influenza infection. Pediatrics 2006; 118: 2409-2417.

40. Bhat $\mathbf{N}$, et al. Influenza-associated deaths among children in the United States, 2003-2004. New England Journal of Medicine 2005; 353: 2559-2567.

41. Grijalva CG, et al. Estimating the undetected burden of influenza hospitalizations in children. Epidemiology and Infection 2007; 135: 951-958.

42. Ji W, et al. The epidemiology of hospitalized influenza in children, a two year population-based study in the People's Republic of China. BMC Health Services Research 2010; 10: 82 .

43. Poehling KA, et al. The underrecognized burden of influenza in young children. New England Journal of Medicine 2006; 355: 31-40.

44. Jefferson T, et al. Vaccines for preventing influenza in healthy children. Cochrane Database of Systematic Reviews 2012; 8: CD004879.

45. Booy R, et al. Cross-reacting antibodies against the pandemic (H1N1) 2009 influenza virus in older Australians. Medical Journal of Australia 2011; 194: 19-23.

46. Hancock K, et al. Cross-reactive antibody responses to the 2009 pandemic H1N1 influenza virus. New England Journal of Medicine 2009; 361: 1945-1952.

47. Miller E, et al. Incidence of 2009 pandemic influenza A $\mathrm{H} 1 \mathrm{~N} 1$ infection in England: a cross-sectional serological study. Lancet 2010; 375: 1100-1108.

48. Hay AJ, et al. The evolution of human influenza viruses. Philosophical Transactions of the Royal Society of London, Series B: Biological Sciences 2001; 356: 1861-1870.
49. Neuzil KM, Bright RA. Influenza vaccine manufacture: keeping up with change. Journal of Infectious Diseases 2009; 200: 835-837.

50. Skowronski DM, et al. Estimating vaccine effectiveness against laboratory-confirmed influenza using a sentinel physician network: results from the 2005-2006 season of dual $\mathrm{A}$ and $\mathrm{B}$ vaccine mismatch in Canada. Vaccine 2007; 25: 2842-2851.

51. Tricco AC, et al. Comparing influenza vaccine efficacy against mismatched and matched strains: a systematic review and meta-analysis. BMC Medicine 2013; 11: 153.

52. Barr IG, et al. WHO recommendations for the viruses used in the 2013-2014 Northern Hemisphere influenza vaccine: Epidemiology, antigenic and genetic characteristics of influenza $\mathrm{A}(\mathrm{H} 1 \mathrm{~N} 1) \mathrm{pdm} 09, \mathrm{~A}(\mathrm{H} 3 \mathrm{~N} 2)$ and $\mathrm{B}$ influenza viruses collected from October 2012 to January 2013. Vaccine 2014; 32: 4713-4725.

53. Regan A, et al. Electronic health record extraction from general practice for monitoring influenza vaccine coverage and effectiveness. Communicable Diseases Control Conference. Brisbane, Australia: Public Health Association of Australasia, 2015, pp. 52.

54. Fielding JE, et al. Estimation of type- and subtypespecific influenza vaccine effectiveness in Victoria, Australia using a test negative case control method, 2007-2008. BMC Infectious Diseases 2011; 11: 170.

55. Fielding JE, et al. Effectiveness of seasonal influenza vaccine against pandemic (H1N1) 2009 virus, Australia, 2010. Emerging Infectious Diseases 2011; 17: 1181-1187.

56. Fielding JE, et al. Moderate influenza vaccine effectiveness in Victoria, Australia, 2011. Eurosurveillance 2012; 17: pii $=20115$.

57. Skowronski D, et al. Interim estimates of influenza vaccine effectiveness in 2012/13 from Canada s sentinel surveillance network, January 2013. Eurosurveillance 2013; 18: pii $=20394$.

58. Skowronski D, et al. Interim estimates of 2013/14 vaccine effectiveness against influenza A(H1N1)pdm09 from Canada s sentinel surveillance network, January 2014. Eurosurveillance 2014; 19.

59. Osterholm MT, et al. Efficacy and effectiveness of influenza vaccines: a systematic review and meta-analysis. Lancet Infectious Diseases 2012; 12: 36-44.

60. Darvishian M, et al. Effectiveness of seasonal influenza vaccine in community-dwelling elderly people: a metaanalysis of test-negative design case-control studies. Lancet Infectious Diseases 2014; 14: 1228-1239.

61. Tamerius J, et al. Global influenza seasonality: reconciling patterns across temperate and tropical regions. Environmental Health Perspectives 2011; 119: 439-445.

62. Tamerius JD, et al. Environmental predictors of seasonal influenza epidemics across temperate and tropical climates. PLoS Pathogens 2013; 9: e1003194.

63. Koul PA, et al. Differences in influenza seasonality by latitude, northern India. Emerging Infectious Diseases 2014; 20: 1746-1749.

64. Australian Government Department of Health. Australian Influenza Surveillance Report. No. 8, 2014, 27 September to 10 October 2014. Canberra: Australian Government Department of Health, 2014. 